\title{
Análisis del e-PEL (Portfolio Europeo de las Lenguas Electrónico): opinión de los alumnos sobre Descriptores, Aprender a aprender y Autoevaluación
}

\section{Analysis of the eELP (Electronic European Language Portfolio): students' opinion about Descriptors, Learning to learn and Self-Assessment}

\author{
Mario-Jesús Mira-Giménez \\ Escuela Oficial de Idiomas de Alicante (España)
}

\section{Resumen}

El presente trabajo analiza el e-PEL (Portfolio Europeo de las Lenguas Electrónico) como aplicación práctica del MCERL (Marco Común Europeo de Referencia para las Lenguas). Se ha pretendido el estudio de sus tres aspectos fundamentales: Descriptores, Aprender a aprender y Autoevaluación. La investigación se ha llevado a cabo durante seis meses, de octubre de 2015 a marzo de 2016 en cuatro grupos de alemán de nivel A2.1, B1.1 y B1.2 de la Escuela Oficial de Idiomas de Alicante, con un total de cien alumnos. La metodología utilizada es cuantitativa y cualitativa: después de la aplicación del e-PEL, los estudiantes han cumplimentado un cuestionario de catorce ítems para averiguar su opinión sobre los tres ámbitos objeto de estudio. Los datos se han analizado de forma global y desagregados por sexo y grupos de edad. Los resultados muestran que los estudiantes valoran positivamente el e-PEL como herramienta de desarrollo de las tres variables analizadas, si bien opinan de forma más favorable sobre la Autoevaluación, mientras que los Descriptores obtienen porcentajes algo inferiores, lo que podría radicar en el cambio de perspectiva en la enseñanza-aprendizaje de lenguas con el estudiante como centro del proceso y en la falta de desarrollo de descriptores del e-PEL. Del estudio de los datos se desprende que sería necesaria una implementación del e-PEL a través de la Web 3.o para adaptarlo a la nueva sociedad plurimodaltic, basada en el aprendizaje continuo y personalizado, la descripción más exhaustiva de los niveles de competencia y más tiempo de adaptación.

Palabras clave: enseñanza de lenguas; aprendizaje en grupo; aprendizaje asistido por ordenador; autonomía; competencias comunicativas.

\section{Abstract}

This paper analyzes the eELP (Electronic European Language Portfolio) as a practical application of the CEFR (Common European Framework of Reference for Languages). The aim has been to study its three main aspects: Descriptors, Learning to learn and Self-Assessment. The research has been conducted for six months from October 2015 to March 2016 in four 
groups of German students (Levels: A2.1, B1.1 and B1.2) at the Official School of Languages in Alicante, on the whole, 100 pupils. The methodology used is quantitative and qualitative: after the application of the eELP, students filled in a questionnaire with fourteen items to find out their opinion on the three areas under study. The data were analyzed globally and broken down by sex and age groups. The results show that students value the eELP positively as a tool for development of the three variables analyzed. Nevertheless, they think more favourably about Self-Assessment, while a bit lower percentages were obtained by Descriptors, what could lie in the change of perspective in language teaching and learning with the student as the centre of the process and the lack of descriptors' development in the eELP. Study data show that an implementation of the eELP through the Web 3.0 would be needed to adapt it to the new plurimodaltic society, based on continuous and personalized learning, as well as a more comprehensive description of competence levels and more time to adapt.

Keywords: language teaching; group learning; computer-assisted learning; autonomy; communicative competences.

Las TIC (Tecnologías de la Información y la Comunicación) por sí solas no cambian los ambientes de aprendizaje, ya que es imprescindible su combinación con estrategias de enseñanza-aprendizaje (Marcinkonienè y Zdanytė, 2016), "que no solo prioricen la adquisición de conocimientos basados en recursos digitales, sino que apoyen un proceso de apropiación de estos conocimientos por parte del alumnado a través de actividades de aprendizaje productivas, experienciales o comunicativas" (García, Yot y Mayor, 2015, p. 119), tal y como propugna el e-PEL (Portfolio Europeo de las Lenguas Electrónico). La Ley Orgánica 8/2013 para la Mejora de la Calidad Educativa (LOMCE) considera también el aprendizaje de los ciudadanos a lo largo de la vida, ya que permite compatibilizar la formación con las obligaciones personales o profesionales.

El e-PEL representa un documento del Consejo de Europa, que promueve la reflexión a través de las muestras que se seleccionan para conformarlo y compartirlo con otros usuarios. Consta de tres secciones: Pasaporte de las Lenguas, Biografía y Dossier:

The Passport shows the learner's competences in different languages and is used by the learner to reflect and provide a self-assessment in terms of different skills (reading, speaking, listening, writing) and by CEFR levels (from A1 to C2). The Biography details linguistic and cultural knowledge and is designed as a guide for planning and evaluating progress 6 through reflection on the learning of languages and cultures, and to help in the formulation of learning plans. The Dossier contains examples of personal work that certify the learner's capacities and linguistic and cultural knowledge (this constitutes the learner's repository of evidence). (LópezFernández, 2014, pp. 5-6) 
Su organización en apartados facilita la sistematización de las experiencias de aprendizaje, que reflejan la consecución de objetivos, teniendo en cuenta el proceso y el resultado. De igual manera, fomenta la aplicación de diferentes estrategias de reflexión, tales como rúbricas, matriz DAFO (Debilidades Amenazas Fortalezas Oportunidades) o estándares específicos (Cacheiro, Sánchez y González, 2016).

\section{MARCO TEÓRICO}

La mayor aportación del e-PEL respecto a su homólogo en formato impreso es el espacio web que constituye un diario digital de aprendizaje a lo largo de la vida (Oner y Adadan, 2016). Una innovación curricular en un contexto determinado no se considera afianzada, hasta que el nuevo método se interioriza y se aplica correctamente en el aula (Rixon, 2016). Quizás por esta razón esta innovación didáctica no ha tenido la repercusión que se le podría presuponer (López Fernández, 2014). La tecnología educativa sirve para transmitir el conocimiento de forma interdisciplinar a través de los diferentes ámbitos en los que se desarrolla el proceso de enseñanza-aprendizaje. Su planificación supone asimismo un aspecto primordial en la formación de los docentes (Sancho, Bosco, Alonso y Sánchez, 2015).

La Resolución de 2 de junio de 2015, de la Secretaría General de Educación aplica el sistema de trabajo del portfolio también a los profesores y a todas las materias, como la Competencia Digital Docente, que abarca cuatro ámbitos: Información, comunicación, creación de contenido, seguridad y resolución de problemas. Dentro de cada bloque, los estándares sirven para implementar dichas competencias y determinan su evaluación de forma análoga a los niveles del MCERL (Marco Común Europeo de Referencia de las Lenguas): usuario principiante: (A1 y A2); usuario medio: (B1 y B2); y usuario avanzado y experto: ( $\mathrm{C} 1$ y C2), en consonancia con las tesis de Komorowska (2016) y Xerri y Campbell (2016).

El portfolio docente requiere un cambio de perspectiva centrado en los procesos de aprendizaje de sus estudiantes, así como la aplicación de estrategias de aprendizaje que fomentan habilidades metacognitivas de orden superior. Asimismo, surge la necesidad de la formación continua (función sumativa) y de información necesaria sobre el proceso (función formativa) (Jurisevic, Enever y Pizorn, 2014). De hecho, este nuevo método se basa en el intercambio de experiencias a través de un diálogo abierto enmarcado en el desarrollo profesional docente, en las competencias profesionales (Denchev, Pavlova y Pavlova, 2016; Xerri y Campbell, 2016). La actualización tecnológica y pedagógica del profesorado de idiomas es primordial, no solo en la formación inicial, sino también en la continua, ya que existe una brecha digital entre los alumnos, que pertenecen a la Generación Z o Generación Tecnológica, y los docentes, en un porcentaje alto, inmigrantes digitales (Faick, 2016; Fernández y Fernández, 2016).

El objetivo de las metodologías, los medios y los recursos radica en promover la motivación del alumnado. La Web 3.0 se entiende como la creación de contenido y 
de servicios de alta calidad producidos por usuarios mediante la tecnología Web 2.0 como plataforma (Calacanis, 2007). La Web 3.0 se caracteriza por la compatibilidad entre sistemas y sus interfaces, integrando plataformas, protocolos y software de código abierto con el fin de crear nuevas herramientas. Otro rasgo significativo es la interoperabilidad en todos los dispositivos, así como el diseño en tres dimensiones y la creación de avatares para interactuar en el mundo virtual. Asimismo, la Web 3.0 supone un control sobre la información, ya que sirve para organizar las aplicaciones, que ofrece la Web 2.o. Por último, esta nueva Web supone una web semántica, puesto que agrega mediante los metadatos significado a la información, por lo que es más fácil acceder y filtrarlos (Santiago y Navaridas, 2012).

Gonzálvez (2011) atribuye a la Web 3.0 en el ámbito educativo la capacidad multimedia, que posibilita el intercambio de documentos en formatos diferentes, la formación de grupos de trabajo de aprendizaje colaborativo y la inclusión de aplicaciones que motivan la comunicación. Asimismo, hace hincapié en la importancia de la Web 3.0 en la mejora de las redes de aprendizaje en lo que respecta al rol del estudiante y la búsqueda de información según el estilo de aprendizaje del alumno.

La Web 3.0 propone nuevos formatos, tales como la iELAO (Enseñanza Integrada de Lenguas Asistida por ordenador) e interfaces con reconocimiento de voz, un portfolio multimedia adaptable y MOOCs (Massive Open Online Course) controlados por el alumno y metodologías para la enseñanza-aprendizaje de lenguas (estilos de aprendizaje, personalización, web semántica). A tales efectos, pone a disposición herramientas como la videoconferencia, las salas virtuales, los whiteboards, los mundos virtuales y las aplicaciones compartidas. Estos cambios se traducen en una nueva relación profesor-alumno, ya que el docente traslada al discente la elección sobre los contenidos, la metodología y las herramientas. El tutor 3.o enseña a aprender, lo que entronca con uno de los pilares del e-PEL como es el aprendizaje estratégico (Sánchez, 2012).

La Web 3.o contribuye, por ejemplo, a través del aprendizaje móvil a la promoción de la autonomía y la adecuación a las necesidades del usuario, lo cual suscita interés, en este caso, por los idiomas, ya que se identifica el aprendizaje con el ocio (Liu y Lan, 2016; Rico y Agudo, 2016):

El e-Learning 2.0 promueve el trabajo en red, los flujos comunicativos multidireccionales y la socialización de la educación y del conocimiento. En la fase de e-Learning 3.0, vinculada también a las herramientas y servicios de la denominada Web 3.0 o web semántica, hablaríamos ya de plataformas de enseñanza/aprendizaje inteligentes personalizables por el propio usuario. (Sotelo, 2009, p.123)

La aplicación del aprendizaje móvil al e-PEL mediante las apps de MALL (Mobile assisted language learning) motivarían el aprendizaje autónomo, ubicuo y asíncrono. No obstante, las necesidades de sincronía se podrían cubrir mediante 
otros canales, tales como chats o microblogging. De esta manera, la implementación del e-PEL a través de su integración en las redes sociales y las apps sería altamente beneficiosa, ya que se fomentaría el aprendizaje social (Barrot, 2016; Jordano, Castrillo y Pareja-Lora, 2015). El e-PEL constituye, por tanto, el nexo entre la Web 3.o, los entornos personales de aprendizaje y la EOI (Escuela Oficial de idiomas) a través de la interacción, generando conocimiento mediante "el aprendizaje múltiple, ubicuo e interrelacionado, que se consigue, media y se construye entre el sistema (la EOI) y el aprendizaje personal" (Mira, 2015, p. 190). El e-PEL requiere una formación previa, tanto para el docente como para el alumnado, de tal manera que todos los actores del proceso interioricen la nueva forma de aprender para adaptar el tiempo disponible al necesario (López, 2016). Se ha probado su validez como innovación educativa para el aprendizaje lingüístico y cultural, a pesar de su escasa repercusión, si bien sería conveniente su implementación tecnológica a través de la Web 3.o (López-Fernández, 2014; Mira, 2016).

El e-PEL constituye una propuesta integrada en la planificación de la enseñanzaaprendizaje, ya que contribuye a detectar necesidades y tomar medidas al respecto, al tiempo que fomenta la autoevaluación a través de la realización de las tareas. Esta forma de trabajo motiva el compromiso respecto a los objetivos de aprendizaje y los criterios de evaluación, ya que pone énfasis en el proceso y el resultado (Cuenca y Lorente, 2015; Sossouvi, 2016), en consonancia con la nueva tendencia evaluadora a nivel europeo:

Sin embargo, en el sistema educativo actual español y europeo se propone que se evalúe todo el proceso educativo de forma objetiva a través de la concreción de los criterios de evaluación por medio de indicadores observables y medibles por tareas: acciones en clase, actitud, trabajo realizado en el día a día tanto en el aula como en casa, etc. y que el examen sea un elemento más a tener en cuenta en la calificación final. (Vázquez, 2016, p. 80)

El e-PEL constituye un instrumento válido para desarrollar la competencia lingüística, intercultural, estratégica y plurilingüe, de manera activa, ya que la recopilación de muestras y la reflexión asociada fomenta la autoevaluación, la revisión de contenidos a través de la toma de conciencia del proceso y el resultado de aprendizaje (Lozano y Giralt, 2014). Desde la perspectiva docente, posibilita una evaluación integral, que también tiene en cuenta los objetivos personales de aprendizaje de cada alumno (Trofimovich, Isaacs, Kennedy, Saito y Crowther, 2016). De hecho, el PEL se fundamenta en principios claves como la autonomía del alumno, el plurilingüismo, el pluriculturalismo, la motivación, la reflexión y las estrategias de aprendizaje (Sævikstrand, 2015).

Las características del ejemplo de aprendizaje, que demuestra la consecución de un descriptor, son dos: el agente (el autor) y el tipo de medio (el formato). Las TIC facilitan la creación y gestión de recursos multimedia en la construcción del aprendizaje (Tan y Liu, 2016), cuya finalidad se refiere a la función que desempeña y 
al descriptor en cuestión. Asimismo, la actividad de aprendizaje implica el patrocinio (el agente) y el tipo de participación (Tur y Urbina, 2016).

El e-PEL posibilita la movilidad laboral y académica gracias a su interoperabilidad, ya que permite su exportación a otras instituciones académicas -en experiencias de movilidad en el EEES (Espacio Europeo de Educación Superior)- o empresas en el mundo laboral globalizado actual (Gil y Roca, 2011; Núñez, 2016; Sevillano y Vázquez, 2015). Asimismo, fomenta la autorregulación del aprendizaje porque la atención se centra principalmente en los estudiantes. Este cambio de perspectiva se plasma en la participación activa de los estudiantes en el proceso a través de la selección y organización de los contenidos, el establecimiento de objetivos y la evaluación del proceso. Del mismo modo, el aprendizaje autorregulado fomenta el desarrollo de la responsabilidad. El e-PEL proporciona a los estudiantes elementos de juicio para detectar puntos fuertes y débiles y tomar medidas al respecto (Yastibas y Yastibas, 2015; Zheng, Liang, Yang y Tsai, 2016).

El desarrollo de la competencia intercultural requiere prácticas de aprendizaje colaborativas, que impliquen la contextualización de las actividades de aprendizaje en comunidades de reflexión, la dualidad de perspectivas de docente y alumno, con el desarrollo de competencias genéricas, las denominadas soft skills, orientadas al desarrollo personal y profesional, de tal manera que se fomente la creación de comunidades críticas y reflexivas (Chuang y Suthers, 2016; McCloskey, 2012). Zheng y otros (2016) recomiendan de hecho que los legisladores tomen conciencia de la influencia negativa que ejercen los exámenes y diversifiquen los métodos de evaluación en la enseñanza-aprendizaje de lenguas. Los portfolios electrónicos como el e-PEL posibilitan formas de evaluación alternativas como las eRúbricas o la coevaluación, que fomentan el desarrollo de competencias generales, que los alumnos necesitan para su desarrollo personal y profesional, donde la autonomía, la interculturalidad y el desarrollo de estrategias desempeñan un papel primordial (Cebrián, Serrano y Ruiz, 2015).

\section{METODOLOGÍA}

Este estudio mixto analiza la aplicación del e-PEL a la enseñanza-aprendizaje de idiomas en la EOI en cuatro grupos de alumnos de alemán de nivel A2.1, B1.1 y B1.2 (primer, tercer y cuarto curso) durante seis meses, de octubre de 2015 a marzo de 2016. El número de alumnos que ha participado en el estudio ha ascendido a cien sujetos. El objetivo de esta investigación consiste en averiguar la opinión de los alumnos respecto a las secciones del e-PEL: descriptores, aprender a aprender y autoevaluación. A tal efecto, se ha realizado un muestreo casual o incidental, seleccionando directa e intencionadamente los individuos de un grupo determinado de alumnos. Para evaluar la representatividad de la muestra se ha comparado la media muestral con la media poblacional de los alumnos de alemán y de los otros idiomas de la EOI en cuanto a sexo y edad con un nivel de confianza del 95\%. La 
recogida de datos se ha llevado a cabo mediante un cuestionario (Anexo 1), en el que las respuestas se han valorado mediante una escala Likert de cuatro puntos. El cuestionario se ha dividido en tres ámbitos: Descriptores (5 ítems), Aprender a aprender (4 ítems) y Autoevaluación ( 5 ítems). Se ha utilizado el alfa de Cronbach para cuantificar el nivel de fiabilidad de la escala de medida para la magnitud inobservable (opinión de los alumnos sobre el aprendizaje mediante el e-PEL) construida a partir de las variables observadas. Los coeficientes de fiabilidad de cada escala son los siguientes:

- Descriptores: 0,77

- Aprender a aprender: 0,85

- Autoevaluación: 0,82

Estos datos confirman la fiabilidad del cuestionario. Asimismo, se ha especificado la fiabilidad de las tres escalas. La muestra de estudio está constituida por cien alumnos, de los cuales $34 \%$ eran hombres y $66 \%$ eran mujeres, porcentajes similares a los alumnos de alemán (29,7\% de hombres y 69,3\% de mujeres) y de los otros diez idiomas (39,4\% de hombres y 60,6\% de mujeres) impartidos en la EOI Alicante. La diferencia estadística entre ambos sexos se puede deber a la mejor capacidad lingüística de las mujeres respecto a los hombres (OCDE, 2015). En lo que respecta a la edad, el 12\% tiene menos de 20 años, el $37 \%$ se sitúa entre 20 y 30 , el $29 \%$ entre 30 y 40 , el $17 \%$ entre 40 y 50 y el $5 \%$ más de 50 . La distribución se puede considerar típica, con concentración en torno a la media y dispersión en los extremos.

\section{ANÁLISIS Y RESULTADOS}

Tabla 1. Descriptores

\begin{tabular}{|l|c|c|c|c|}
\hline & Nada (o) & Poco (1) & Bastante (3) & Mucho (4) \\
\hline Comprensión escrita & $4 \%$ & $13 \%$ & $46 \%$ & $37 \%$ \\
\hline Comprensión oral & $\mathbf{1 1 \%}$ & $\mathbf{1 9 \%}$ & $36 \%$ & $34 \%$ \\
\hline Expresión escrita & $\mathbf{3 \%}$ & $\mathbf{2 2 \%}$ & $43 \%$ & $32 \%$ \\
\hline Expresión oral & $\mathbf{1 1 \%}$ & $\mathbf{2 2 \%}$ & $39 \%$ & $\mathbf{2 8 \%}$ \\
\hline Interacción oral & $\mathbf{9 \%}$ & $31 \%$ & $31 \%$ & $\mathbf{2 9 \%}$ \\
\hline
\end{tabular}

Fuente: Cuestionario de los estudiantes.

Elaboración propia.

Agregados los datos, la moda en todas las variantes es 3 (Bastante), mientras que en Comprensión oral, la variable Mucho registra un 34\%, en cambio en Interacción oral, Poco y Bastante saturan con un 31\%. Por consiguiente, los alumnos consideran 
que el e-PEL ha contribuido al desarrollo de destrezas a través de los descriptores en todas las variables, con una varianza del $23 \%$ (83\% en Comprensión escrita y $60 \%$ en Interacción oral). La opinión de los alumnos sobre el e-PEL es peor en las destrezas orales que en las escritas: la respuesta Nada registra respectivamente $11 \%$ y $5,3 \%$ de promedio. Esta tendencia también se refleja en la proporción de los alumnos que responden Mucho: el porcentaje en Comprensión escrita, Comprensión oral y Expresión escrita es ligeramente mayor que en Expresión oral e Interacción oral.

$\mathrm{Si}$ desagregamos por edad, continúa predominando en todos los grupos Bastante, excepto en el grupo de menos de 20 años, donde también satura Poco (53\%). Hay que destacar que satura Mucho con un 92\% entre los mayores de 50 años en Comprensión Oral y Comprensión Escrita. Desagregados los datos por sexo, las mujeres consideran que el e-PEL ha contribuido más al desarrollo de descriptores que los hombres, ya que satura la variable Mucho en Comprensión escrita y Expresión escrita con 46\%, mientras que en los hombres satura Poco en Interacción oral con 42\%. En el resto de variables predomina Bastante. Es importante destacar que ninguna mujer tiene una opinión muy negativa sobre el e-PEL en las variables Comprensión escrita y Expresión escrita.

Si desagregamos los resultados por sexo, divergen de manera notable: $61 \%$ entre las mujeres, $73 \%$ en los hombres $(\mathrm{C}=0,074$, Sig.=0,318). Sin embargo, los porcentajes por edad, no difieren tanto: $61 \%$ en los menores de 20 años, el $71 \%$ entre 20 y 30 años, el $64 \%$ entre 30 y $40,63 \%$ entre 40 y 50 y $59 \%$ entre los mayores de $50(\mathrm{C}=0,123$, Sig. $=0,263)$. El valor de las pruebas KMO (Kaiser, Meyer y Olkin) y de esfericidad de Bartlett ascienden respectivamente a 0,77 y 471 con un nivel de significación de 0,000 .

Tabla 2. Aprender a aprender

\begin{tabular}{|l|c|c|c|c|}
\hline & Nada (0) & Poco (1) & Bastante (3) & Mucho (4) \\
\hline Contenidos nuevos & $\mathbf{2 \%}$ & $\mathbf{1 8 \%}$ & $40 \%$ & $40 \%$ \\
\hline Organización & $\mathbf{0} \%$ & $\mathbf{2 1 \%}$ & $34 \%$ & $45 \%$ \\
\hline Reflexión & $5 \%$ & $\mathbf{2 4 \%}$ & $39 \%$ & $32 \%$ \\
\hline Solución de problemas & $\mathbf{4} \%$ & $\mathbf{1 9 \%}$ & $41 \%$ & $36 \%$ \\
\hline
\end{tabular}

Fuente: Cuestionario de los estudiantes.

Elaboración propia.

Agregados los resultados, la respuesta que predomina en las variables Reflexión y Solución de problemas es Bastante (39\% y 41\%, respectivamente), mientras que en Organización satura Mucho (45\%) y en Contenidos nuevos, Bastante y Mucho (40\%). El porcentaje de alumnos que valora positivamente la variable Aprender a aprender oscila del $71 \%$ al $80 \%$. Por tanto, la opinión de los alumnos sobre el e-PEL mejora respecto al desarrollo de destrezas a través de los descriptores. Reseñable es que ningún alumno opina que el e-PEL no contribuye en absoluto a la organización. 
Las variables con resultados algo más negativos son Reflexión (29\%) y Solución de problemas (23\%).

Desagregados los resultados por edad, satura en la mayoría de grupos Bastante. No obstante, predomina en el grupo de menos de 20 años, Poco (91\%) en Reflexión, Organización y Solución de Problemas. Asimismo, hay que reseñar que satura Mucho en el grupo de 20-30 años (42\%) en Organización y en el grupo de 30-40 años en Reflexión con un 50\%. Si desagregamos por sexo, en las mujeres satura Mucho en Contenidos nuevos y Organización, con respectivamente 46\%, mientras que en los hombres predomina Bastante en todas las variables, si bien los porcentajes son algo menores: 42\% en Contenidos nuevos y 28\% en Organización.

Si disociamos los datos por sexo, los resultados son más similares que en la variable Descriptores: mujeres (68\%) y hombres (67\%) (C=0,067, Sig. $=0,223)$. No obstante, los porcentajes por edad presentan mayor variabilidad: $59 \%$ en los menores de 20 años, el $73 \%$ entre 20 y 30 años, el $70 \%$ entre 30 y $40,65 \%$ entre 40 y 50 y $58 \%$ entre los mayores de $50(\mathrm{C}=0,134, \mathrm{Sig} .=0,289)$. El valor de las pruebas KMO y de esfericidad de Bartlett ascienden respectivamente a 0,73 y 506 con un nivel de significación de o,ooo.

Tabla 3. Autoevaluación

\begin{tabular}{|l|c|c|c|c|}
\hline & Nada (0) & Poco (1) & Bastante (3) & Mucho (4) \\
\hline Comprensión escrita & $4 \%$ & $21 \%$ & $41 \%$ & $34 \%$ \\
\hline Comprensión oral & $\mathbf{1 1 \%}$ & $\mathbf{2 6 \%}$ & $34 \%$ & $29 \%$ \\
\hline Expresión escrita & O\% & $14 \%$ & $41 \%$ & $45 \%$ \\
\hline Expresión oral & O\% & $14 \%$ & $34 \%$ & $52 \%$ \\
\hline Interacción oral & O\% & $21 \%$ & $29 \%$ & $50 \%$ \\
\hline
\end{tabular}

Fuente: Cuestionario de los estudiantes.

Elaboración propia.

Agregados los datos, la moda en Comprensión escrita y Comprensión oral es 3 (Bastante), mientras que en el resto de variables es 4 (Mucho). En consecuencia, el aspecto del e-PEL que los alumnos valoran más es la Autoevaluación, especialmente en las destrezas orales con porcentajes entre el $45 \%$ y el $52 \%$. De hecho, las opiniones positivas oscilan entre el $63 \%$ y el $86 \%$. En estas destrezas, destaca que ninguno de los sujetos opina muy negativamente. En consecuencia, la varianza es menor.

Si desagregamos por edad, continúa predominando en todos los grupos Mucho, excepto en el grupo de menos de 20 años, donde satura Bastante (49\%). Hay que reseñar que predomina la respuesta Mucho con un 100\% entre los mayores de 50 años en Comprensión Oral y Comprensión Escrita. Desagregados los datos por sexo, tanto hombres como mujeres consideran que el e-PEL constituye una herramienta válida de autoevaluación, si bien satura Bastante en Comprensión escrita en los hombres (57\%) y en Comprensión oral entre las mujeres (38\%). 
Si desagregamos los resultados por sexo, los resultados son similares: (64\%) mujeres, (63\%) hombres $(\mathrm{C}=0,067$, Sig. $=0,243)$. Los porcentajes por edad tampoco presentan divergencias notables: $62 \%$ en los menores de 20 años, el $69 \%$ entre 20 y 30 años, el $70 \%$ entre 30 y $40,64 \%$ entre 40 y 50 y $63 \%$ entre los mayores de $50(\mathrm{C}=0,134$, Sig. $=0,287)$. El valor de las pruebas KMO y de esfericidad de Bartlett ascienden respectivamente a 0,79 y 466 con un nivel de significación de 0,000.

\section{DISCUSIÓN Y CONCLUSIONES}

De los resultados, se desprende globalmente que los alumnos valoran positivamente el e-PEL como herramienta para el desarrollo de las tres variables estudiadas: Descriptores (71\%), Aprender a aprender (76,75\%) y Autoevaluación $(77,8 \%)$, resultados en consonancia con otras investigaciones similares sobre portfolios electrónicos aplicados al aprendizaje de lenguas (Bolliger y Shepherd, 2010; Castañeda y Rodríguez, 2011; Wang y Chang, 2010) y de estudios específicos del e-PEL (Mira, 2016). En lo que respecta al Desarrollo de descriptores, los discentes opinan que la contribución a las destrezas escritas es mayor que a las destrezas orales. Este hecho podría estar motivado por la dificultad que entrañan la comprensión, la expresión y la interacción oral sin un entorno de inmersión lingüística (Anderson, 2016). Esta hipótesis se confirma con la media de calificaciones de este grupo de alumnos del curso anterior (Comprensión escrita: 72,29\%; Expresión escrita: 69,45\%; Comprensión oral: 62,78\%; Expresión e Interacción oral: 63,38\%). En consecuencia, sería conveniente implementar el e-PEL con la Web 3.0, de tal manera que el sistema seleccionara los perfiles de los intercambios lingüísticos online, que mejor correspondieran al usuario, como ofrece el portfolio electrónico Mahara (Godwin, 2016). Las mujeres valoran más positivamente la comprensión y la expresión escrita que los hombres, lo cual podría derivar de sus mejores resultados en las destrezas escritas en el Informe PISA (OCDE, 2015).

En lo referente a la variable Aprender a aprender, la opinión de los alumnos respecto a Organización y Contenidos nuevos es más favorable que sobre las variables Reflexión y Solución de problemas. Este hecho podría estar motivado porque las dos primeras variables hacen referencia al primer estadio de la metodología del e-PEL en lo que respecta a planificación del aprendizaje y asimilación de nuevas estructuras y vocabulario, mientras que las dos últimas requieren un nivel de abstracción mayor, para poder reflexionar sobre los problemas encontrados y sus soluciones. Por consiguiente, sería necesario más tiempo y práctica para asumir esta nueva forma de aprendizaje, en consonancia con Coelho, Oller y Serra (2015).

La variable Autoevaluación es la que registra mejores resultados, en especial en las destrezas productivas, lo cual podría estar motivado por el mayor grado de subjetividad que presentan y la dificultad que reviste detectar las necesidades de aprendizaje, si bien el desarrollo de descriptores del e-PEL es parcial. Por tanto, sería conveniente especificar los objetivos de aprendizaje al máximo en aras de 
una descripción exhaustiva del nivel de competencia, según las recomendaciones de Llorián (2016). Los estudiantes mantienen un registro escrito de sus reflexiones a través, por ejemplo, de un diario de aprendizaje, que puede servir de evaluación sumativa. La plataforma del SEPIE (Servicio Español para la Internacionalización de la Educación) podría ofrecer una función de búsqueda de usuarios y tareas para llevar a cabo proyectos telecolaborativos, materiales de formación y manuales de apoyo para los educadores, de acuerdo con Butler (2016) y Helm y Guth (2016). Por consiguiente, el e-PEL constituiría una herramienta de aprendizaje y evaluación de tres competencias básicas: lingüística, intercultural y digital.

La filosofía del e-PEL aplicada a la función de profesor entronca con el modelo TPACK (Technological, Pedagogical and Content Knowledge), como marco de referencia entre la formación del profesorado y la integración efectiva de las TIC (Roig, Mengual y Quinto, 2015), materializándose en el portfolio docente, cuya actualización tecnológica sería primordial (portfolio electrónico docente), ya que hasta ahora todos los modelos están en formato impreso.

En conclusión, se podría afirmar que el e-PEL supone el vínculo entre el enfoque teórico del MCERL (Marco Común Europeo de Referencia para las Lenguas) (Consejo de Europa, 2002) y la Web 3.0 aplicada al aprendizaje de lenguas en el seno de una sociedad plurimodaltic (Sevillano, Quicios y González, 2016), porque las TIC han convertido el aprendizaje permanente en continuo y el enfoque del e-PEL las utiliza para personalizarlo y adaptarlo al aprendiente, de acuerdo con las tesis de Maina y García (2016).

\section{REFERENCIAS BIBLIOGRÁFICAS}

Anderson, J. (2016). The Critical Connections pedagogical framework. En J. Anderson y V. Macleroy (Eds.), Multilingual Digital Storytelling (226-247). London: Routledge.

Barrot, J. S. (2016). Using Facebook-based e-portfolio in ESL writing classrooms: impact and challenges. Language, Culture and Curriculum, 1-16. doi:10.108 o/07908318.2016.1143481

Butler, Y. G. (2016). Self-assessment of and for young learners' foreign language learning. En M. Nikolov (Ed.), Assessing Young Learners of English: Global and Local Perspectives (291-315). Springer International Publishing.

Cacheiro González, M. L., Sánchez Romero, C., y González Lorenzo, J. M. (2016).
Recursos tecnológicos en contextos educativos. Madrid: Editorial UNED.

Calacanis, J. (2007). Web 3.0, la definición oficial. Recuperado de http://www.faqmac.com/reportajes/web-30-definicionoficial-jason-calacanis/25512

Cebrián, M. Serrano Angulo, J., y Ruiz Torre, M. (2015). Las eRúbricas en la evaluación cooperativa del aprendizaje en la Universidad [eRubrics in Cooperative Assessment of Learning at University]. Comunicar, 43 (XXII), 153-161. doi: http://dx.doi.org/10.3916/C43-2014-15

Chuang, L. M. L., y Suthers, D. D. (2016). Behavioral Manifestations of Intercultural Competence in Computer-Mediated Intercultural Learning. En 2016 49th Hawaii International Conference on 
System Sciences (HICSS) (pp. 20852094). IEEE.

Coelho, E., Oller, J., y Serra, J. M. (2015). Una propuesta de adaptación del Marco Europeo para los ESL. Bellaterra: journal of teaching and learning language and literature, 8(1), 10-27. doi: http://dx.doi. org/10.5565/rev/jtl3.612

Consejo de Europa (2002). Marco común europeo de referencia para las lenguas. Madrid: Ministerio de Educación, Cultura y Deporte, Subdirección General de Cooperación Internacional, Secretaría General Técnica del MECD-Subdirección General de Información y Publicaciones y Grupo ANAYA, SA.

Cuenca, B., y Lorente, P. (2015). El portfolio electrónico: Un trabajo "con vistas". Alternativas para evaluar y reflexionar en los cursos de lengua. En N. Ibarra Rius, J. Ballester Roca, M. L. Carrió Pastor y F. Romero Forteza (Eds.), Retos en la adquisición de las literaturas y de las lenguas en la era digital (181195). Valencia: Editorial Universidad Politécnica de Valencia.

Denchev, S., Pavlova, I., y Pavlova, M. (2016). The Reform of Higher Education through Alternative University Teaching Models. Creative Education, 7, 302-306. doi: $10.4236 /$ ce.2016.72020

Faick, M. (2016). Pensar, reflexionar y cambiar: la experiencia del portafolio docente. Revista UNAH INNOV@, 2, 13-22. doi: http://dx.doi.org/10.5377 unahinnov.voi2.2372

Fernández Cruz, F. J., y Fernández Díaz, M. J. (2016). Los docentes de la Generación Z y sus competencias digitales [Generation Z's Teachers and their Digital Skills]. Comunicar: Revista científica iberoamericana de comunicación $y$ educación, 24(46), 97-105. doi: http:// dx.doi.org/10.3916/C46-2016-10

García, C. M., Yot Domínguez, C., y Mayor Ruiz, C. (2015). Enseñar con tecnologías digitales en la universidad [University
Teaching with Digital Technologies]. Comunicar: Revista Científica de Comunicación y Educación, 45(23), 117124. doi: http://dx.doi.org/10.3916/C452015-12

Gil Serra, A. F., y Roca Piera, J. (2011). Movilidad virtual, reto del aprendizaje de la educación superior en la Europa 2020. Revista de Educación a Distancia, 26, 1-16. Recuperado de http://www.um.es ead/red/26/gil roca.pdt

Godwin Jones, R. (2016). Integrating Technology into Study Abroad. Language Learning \& Technology, 2O(1), 1-20. Recuperado de http://llt.msu.edu/issues/ february2016/emerging.pdif

Gonzálvez Vallés, J. E. (2011). La Web 2.0 y 3.o en su relación con el EEES. Madrid: Editorial Visión Libros.

Helm, F., y Guth, S. (2016). Telecollaboration and language learning. En F. Farr y L.Murray, The Routledge handbook of language learning and technology (240254). Nueva York: Routledge

Jordano, M., Castrillo, M. D., y Pareja Lora, A. (2015). El aprendizaje de lenguas extranjeras mediante tecnología móvil en el contexto de la educación a distancia y combinada. RIED. Revista Iberoamericana de Educación a Distancia, 19(1), 25-40. doi: http:// dx.doi.org/10.5944/ried.19.1.1528

Jurisevic, M., Enever, J., y Pizorn, K. (2014). Triple Tool Effect: Professional Portfolios in Teaching Foreign Languages. Porta Linguarum: revista internacional de didáctica de las lenguas extranieras, 21, 7-24. Recuperado de http://www. ugr.es/ portalin/articulos/PL numero21/1\%20\%20Mojca.pdt

Komorowska, H. (2016). The European Language Policy and the teaching profession in the $21^{\text {st }}$ century. Studia Linguistica Universitatis Iagellonicae Cracoviensis, 2015(3), 135-150. doi: 10.4467/20834624SL.15.014.3935 
Liu, S. H. J., y Lan, Y. J. (2016). Social Constructivist Approach to Web-Based EFL Learning: Collaboration, Motivation, and Perception on the Use of Google Docs. Journal of Educational Technology \& Society, 19(1), 171-186. Recuperado de http://www.ifets.info/journals/19 1/15. pdf

Llorián, S. (2016). La descripción empírica de los niveles de referencia del español a partir de corpus de aprendientes. Revista Nebrïa de Lingüística Aplicada, 2016(20). Recuperado de http://www. nebrija.com/revista-linguistica/ladescripcion-empirica-de-los-niveles-dereferencia-del-espa\%C3\%B1ol-a-partirde-corpus-de-aprendientes

López Berrio, J. J. (2016). Una experiencia de aprendizaje basado en proyectos de alto contenido tecnológico. Revista Tecnología, Ciencia y Educación, 3, 4361. Recuperado de http://revistasocitec. org/index.php/TCE/article/view/64/62

López Fernández, O. (2014). University teaching experience with the electronic European Language Portfolio: an innovation for the promotion of plurilingualism and interculturality [Experiencia docente universitaria con el Portfolio Europeo de Lenguas electrónico: una innovación para la promoción del plurilingüismo y la interculturalidad]. Cultura y Educación, 26(1), 211-225. doi: 10.1080/11356405.2014.908667

Lozano, R. A., y Giralt Lorenz, M. (2014). El porfolio digital y la comunicación multimodal en el aprendizaje de la lengua oral: estudio piloto de un caso. En L. P. Cancelas, R. Jiménez-Fernández, M. F. Romero Oliva y S. Sánchez Rodríguez (Coords.), Una educación lingüística y literaria en el siglo XXI (1-11). Granada: Grupo Editorial Universitario.

Maina, M. F., y García González, I. (2016). Articulating Personal Pedagogies Through Learning Ecologies. En B. Gros y M. Maina, The Future of Ubiquitous
Learning (73-94). Berlin/Heidelberg: Springer.

Marcinkonienè, R., y Zdanytè, J. (2016). Screen vs Paper in Foreign Language Learning. Studies About Lanquages, 27, 106-114. doi: http://dx.doi.org/10.5755 j01.sal.0.27.13748

McCloskey, E. M. (2012). Docentes globales: un modelo para el desarrollo de la competencia intercultural on-line [Global Teachers: AConceptual Model for Building Teachers' Intercultural Competence Online]. Comunicar, 38(XIX), 41-49. doi: http://dx.doi.org/10.3916/C38-2012-02 04

Mira Giménez, M. J. (2015). PLE y E-PEL para el aprendizaje de idiomas: similitudes y diferencias. $3 C$ TIC, $4(3), 185-196$. doi: http://dx.doi.org/10.17993/3ctic

Mira Giménez, M. J. (2016). Portfolio Europeo de las Lenguas Electrónico: una experiencia de innovación en la Escuela Oficial de Idiomas. EDUTEC Revista Electrónica de Tecnología Educativa, 55, 1-26. Recuperado de http://www.edutec. es/revista/index.php/edutec-e/article/ view/644/Edutec n55 Mira

Núñez Cortés, J. A. (2016). Alfabetización académica y competencia comunicativa en educación superior. Revista UNAH INNOV@, 3,16-20. doi: http://dx.doi. org/10.5377/unahinnov.voi3.2381

OCDE (2015). PISA in Focus. Recuperado de http://www.oecd.org/pisa/pisaproducts/ pisainfocus/PIF-49\%20\%28esp\%29.pdf

Oner, D., y Adadan, E. (2016). Are integrated portfolio systems the answer? An evaluation of a web-based portfolio system to improve preservice teachers' reflective thinking skills. Journal of Computing in Higher Education, 1-25. doi: 10.1007/ s12528-016-9108-y

Resolución de 2 de junio de 2015, de la Secretaría General de Educación, por la que se publica el Portfolio de Competencia Digital Docente de Extremadura. DOE, 112, de 12 de junio de 2015 . 
Rico García, M. M. y Agudo Garzón, J. E. (2016). Aprendizaje móvil de inglés mediante juegos de espías en Educación Secundaria. RIED: Revista Iberoamericana de Educación a Distancia, 19(1), 121-139. Recuperado de http://revistas.uned.es/index.php/ried/ article/view/14893/13571

Rixon, S. (2016). Do developments in assessment represent the 'coming of age'of young learners English language teaching initiatives? The international picture. En M. Nikolov (Ed.), Assessing Young Learners of English: Global and Local Perspectives (19-41). Springer International Publishing.

Roig Vila, R., Mengual Andrés, S., y Quinto Medrano, P. (2015). Conocimientos tecnológicos, pedagógicos y disciplinares del profesorado de Primaria [Primary Teachers' Technological, Pedagogical and Content Knowledge]. Comunicar, 45 (XXIII), 151-159. doi: http://dx.doi. org/10.3916/C45-2015-16

Sævikstrand, B. (2015). El Portfolio Europeo de las Lenguas en la enseñanza de español de la secundaria noruega. Una investigación-acción. Recuperado de http://bora.uib.no/bitstream/ handle/1956/10608/138373954. pdf?sequence $=1$ \&isAllowed $=y$

Sánchez Arán, I. (2012). El futuro de la enseñanza y aprendizaje de lenguas extranjeras mediante las TIC: web $3.0 \mathrm{y}$ e-learning 3.0 Recuperado de https:// prezi.com/n2gdx4klfker/el-futuro-dela-ensenanza-y-aprendizaje-de-lenguasextranjeras-mediante-las-tic-web-30-y-elearning-30/

Sancho Gil, J. M., Bosco, A., Alonso Cano, C., y Sánchez, J. A. (2015). Formación del profesorado en Tecnología Educativa: de cómo las realidades generan los mitos. RELATEC: Revista Latinoamericana de Tecnología Educativa, 14(1), 17-30. doi: 10.17398/1695288X.14.1.17
Santiago Campión, R., y Navaridas Nalda, F. (2012). La web 2.0 en escena. Pixel-Bit: Revista de medios y educación, 41, 19-30. Sevillano García, M. L., y Vázquez Cano, $\mathrm{E}$ (2015). Modelos de investigación en contextos ubicuos y móviles en Educación Superior. Madrid: MC Graw Hill.

Sevillano García, M., Quicios García, M., y González García, J. (2016). Posibilidades ubicuas del ordenador portátil: percepción de estudiantes universitarios españoles [The Ubiquitous Possibilities of the Laptop: Spanish University Students' Perceptions]. Comunicar, 46(24), 8795. doi: http://dx.doi.org/10.3916/C462016-og

Sossouvi, L. F. (2016). Enseñanza y aprendizaje del ELE en Benín: ¿qué puede aportar la adaptación del MCER? [Teaching and learning of SFL in Benin: What can provide the adaptation of the CEFR?]. Tejuelo, 23(1), 36-62. doi: 10.17398/1988-8430.23.1.36

Sotelo González, J. (2009). Del” e-learning” al" m-learning": una academia en cada" iPhone". Telos: Cuadernos de comunicación e innovación, 81, 122-128.

Tan, C., y Liu, M. (2016). Using ICT in Teaching the Chinese Language: Practices and Reflections from Singapore. En K. Soh (Ed.), Teaching Chinese Language in Singapore (45-63). Springer Singapore.

Trofimovich, P., Isaacs, T., Kennedy, S., Saito, K., y Crowther, D. (2016). Flawed self-assessment: Investigating self- and other-perception of second language speech. Bilingualism: Language and Cognition, 19(1), 122-140. doi: http:/ dx.doi.org/10.1017/S1366728914000832

Tur Ferrer, G., y Urbina Ramírez, S. (2016). Rúbrica para la evaluación de portafolios electrónicos en el entorno de la web social. Pixel-Bit: Revista de medios y educación, 48, 83-96. doi: 0.12795/pixelbit.2016. i48.06

Vázquez Cano, E. (2016). El derecho conculcado del alumno a una evaluación 
objetiva en la LOMCE. REJIE: Revista Jurídica de Investigación e Innovación Educativa, 13, 76-92. Recuperado de http://www.eumed.net/rev/rejie/13/ lomce.pdf

Xerri, D., y Campbell, C. (2016). E-portfolios in teacher development: the better option? ELT Journal. doi: 10.1093/elt/ccw032

Yang, J. H. (2016). Focus on Australian English: a critical learning portfolio pedagogy. Language, Culture and Curriculum, 29(2), 1-22. doi: 10.1080/07908318.2015.1128948

Yastibas, A. E., y Yastibas, G. C. (2015). The use of e-portfolio-based assessment to develop students' self-regulated learning in English language teaching. ProcediaSocial and Behavioral Sciences, 176, 3-13. doi: 10.1016/j.sbspro.2015.01.437

Zheng, C., Liang, J. C., Yang, Y. F., y Tsai, C. C. (2016). The relationship between Chinese university students' conceptions of language learning and their online self-regulation. System, 57, 66-78. doi:10.1016/j.system.2016.01.005. Recuperado de http://www. sciencedirect.com/science/article/pii/ S0346251X16000063

\section{PERFIL ACADÉMICO Y PROFESIONAL DEL AUTOR}

Mario Jesús Mira Giménez es licenciado en Traducción e Interpretación (Inglés) (Universidad de Alicante) y cursó el Máster en Estudios Europeos (Universidad de Leipzig) y el Doctorado en TIC aplicadas a la Enseñanzay Tratamiento de Lenguas (UNED). Ha realizado su tesis doctoral sobre portfolios electrónicos aplicados al aprendizaje de idiomas. Desde 2004 trabaja como profesor de alemán en la Escuela Oficial de Idiomas de Alicante y desde 2013 es el coordinador TIC. E-mail: mariojmira@gmail.com

\section{DIRECCIÓN DEL AUTOR}

Escuela Oficial de Idiomas de Alicante

C/Marqués de Molins, 56-58

03004 Alicante (España)

Fecha de recepción del artículo: 08/05/2016

Fecha de aceptación del artículo: 27/06 /2016

\section{Como citar este artículo:}

Mira Giménez, M. J. (2017) Análisis del e-PEL (Portfolio Europeo de las Lenguas Electrónico): opinión de los alumnos sobre Descriptores, Aprender a aprender y Autoevaluación. RIED. Revista Iberoamericana de Educación a Distancia, 2o(1), pp. 207-222. doi: http://dx.doi.org/10.5944/ried.20.1.16519 


\section{ANEXO 1: Cuestionario}

Después del trabajo con el e-PEL, valora los aspectos siguientes según la contribución del e-PEL a su desarrollo. Haz una cruz en la categoría que representa tu opinión:

\begin{tabular}{|l|l|l|l|l|}
\hline \multicolumn{2}{|c|}{ Nada (0) } & \multicolumn{1}{|c|}{ Poco (1) } & Bastante (3) & Mucho (4) \\
\hline $\begin{array}{l}\text { 1. Descriptores de } \\
\text { comprensión escrita }\end{array}$ & & & \\
\hline $\begin{array}{l}\text { 2. Descriptores de } \\
\text { comprensión oral }\end{array}$ & & & \\
\hline $\begin{array}{l}\text { 3. Descriptores de } \\
\text { expresión escrita }\end{array}$ & & & \\
\hline $\begin{array}{l}\text { 4. Descriptores de } \\
\text { expresión oral }\end{array}$ & & & \\
\hline $\begin{array}{l}\text { 5. Descriptores de } \\
\text { interacción oral }\end{array}$ & & & \\
\hline $\begin{array}{l}\text { 6. Contenidos nuevos } \\
\text { 7. Organización }\end{array}$ & Aprender a aprender & & \\
\hline $\begin{array}{l}\text { 8. Reflexión } \\
\text { 9. Solución de } \\
\text { problemas }\end{array}$ & & & & \\
\hline $\begin{array}{l}\text { 10.Autoevaluación de la } \\
\text { comprensión escrita }\end{array}$ & & & & \\
\hline $\begin{array}{l}\text { 11. Autoevaluación de la } \\
\text { comprensión oral }\end{array}$ & & & & \\
\hline $\begin{array}{l}\text { 12. Autoevaluación de la } \\
\text { expresión escrita }\end{array}$ & & & & \\
\hline $\begin{array}{l}\text { 13. Autoevaluación de la } \\
\text { expresión oral }\end{array}$ & & & & \\
\hline $\begin{array}{l}\text { 14. Autoevaluación de la } \\
\text { interacción oral }\end{array}$ & & & & \\
\hline
\end{tabular}

